\title{
Diffuse Midline Glioma (H3K27M mutant) in Adult: A Diagnostic Challenge
}

\author{
Sonal Paul, Nitin.M. Gadgil and Chetan Chaudhari
}

Department of Pathology, Lokmanya Tilak Medical college and General Hospital, Sion, Mumbai

\section{ABSTRACT}

Introduction: Diffuse midline glioma (DMG), H3K27M mutant is an infiltrative midline high grade glioma with predominantly astrocytic differentiation and K27M mutation in either H3F3A or HIST1H3B/C.

Case Report: A45-year-old female presented with complaints of headache and memory loss for 3 months. MRI was suggestive of an infiltrative mass lesion in the quadrigeminal plate cistern suggestive of pineal neoplasm. Squash and histomorphology showed a low-grade astrocytic tumour with infiltrative growth pattern. Microvascular proliferation and necrosis were absent. Immunohistochemistry showed loss of ATRX protein, focal positivity for p53 proteinand IDH1R132H negativity.On molecular analysis, H3K27M mutation was noted and the case was labelled as DMG H3K27Mmutant (WHO IV)

Conclusion: DMG (H3K27M) is a newly added entity in the WHO 4th revised editionof 2016. It presents with a diagnostic challenge as it hasvariedhistomorphology,not requiring atypia, mitosis, endothelial hyperplasia and necrosis for diagnosis as Grade IV.

\section{Keywords: Adult, Diffuse Midline Glioma,H3K27M Mutation}

\section{Introduction}

The 2016 WHO Classification has combined histological as well as molecular parameters as a pre requisite before rendering the diagnosis of any CNS tumor as opposed to the earlier classifications which were based primarily on morphology. Revised 2016 WHO Classification has recognized Diffuse midline glioma (DMG) (H3K27M mutant) as a distinct entity corresponding to WHO grade IV. Previously, these cases were classified as brainstem gliomas or Diffuse intrinsic pontine gliomas. They have an aggressive clinical behaviour and distinct molecular features ${ }^{[1]} \mathrm{It}$ is usually seen in children but can also be seen in adults with a median age of 32 years and a slight female predominance. ${ }^{[2]}$

\section{Case Report}

We present a 45-year-old female with complaints of headache and memory loss for 3 months. MRI was suggestive of an infiltrating mass $3 \times 3 \times 2 \mathrm{~cm}$, hyper intense on $\mathrm{T} 2$ with epicentre in quadrigeminal plate cistern below the splenium and above the cerebellum and vein of Galen in theposterior mid brain region extending to the pineal region. Radiology favored a pineal neoplasm. [fig.1] A surgical resection of the tumour was done and intra operative squash [fig.2a] showed a low-grade tumour with spindle cell morphology. Subsequently on histopathology an astrocytic tumour with infiltrative growth pattern and a nodular lobular (plexiform) architecture, with moderate nuclear pleomorphism, few large nuclei and calcification was seen. No microvascular proliferation or necrosis was seen. [fig 2b,2c.2d] Special stain for reticulin confirmed the nodular architecture. Immunohistochemistry was performed which showed CD34 negativity suggestive of no vascular proliferation. Loss of ATRX protein[fig.3a], focal positivity for p53 protein [fig.3b], low MIB-1 index (3-4\%) [fig.3c] and IDH1R132H negativity [fig.3d] was noted. A diagnosis of pilocytic astrocytoma was considered on the basis of histomorphology and immunohistochemistry.

However, in view of the midline location and aggressive clinical behaviour, a molecular analysis was performed. Template DNA isolated from the tumor cells was amplified via Polymerase Chain Reaction using H3F3A primers $(0.8 \mu \mathrm{M})$ flanking a 300-base pair exonal region encoding Lys27 and Gly34 in Histone H3.3. Gene sequencing for histone was carried out by Sanger sequencing, which showed aLysine (AAG) to methionine (ATA) substitution at codon 27 in Histone 3A (H3F3A) resulting in H3K27M mutation. [fig.4] Specific antibody against $\mathrm{H} 3 \mathrm{~K} 27 \mathrm{M}$ mutation was not used. Integrated diagnosis of Diffuse midline glioma H3K27M (WHO grade 4) was made. The patient expired within a month following surgery.

\section{Discussion}

Adult brainstem gliomas account for $<2 \%$ of the adult gliomas. Their radiographic appearance varies widely with 


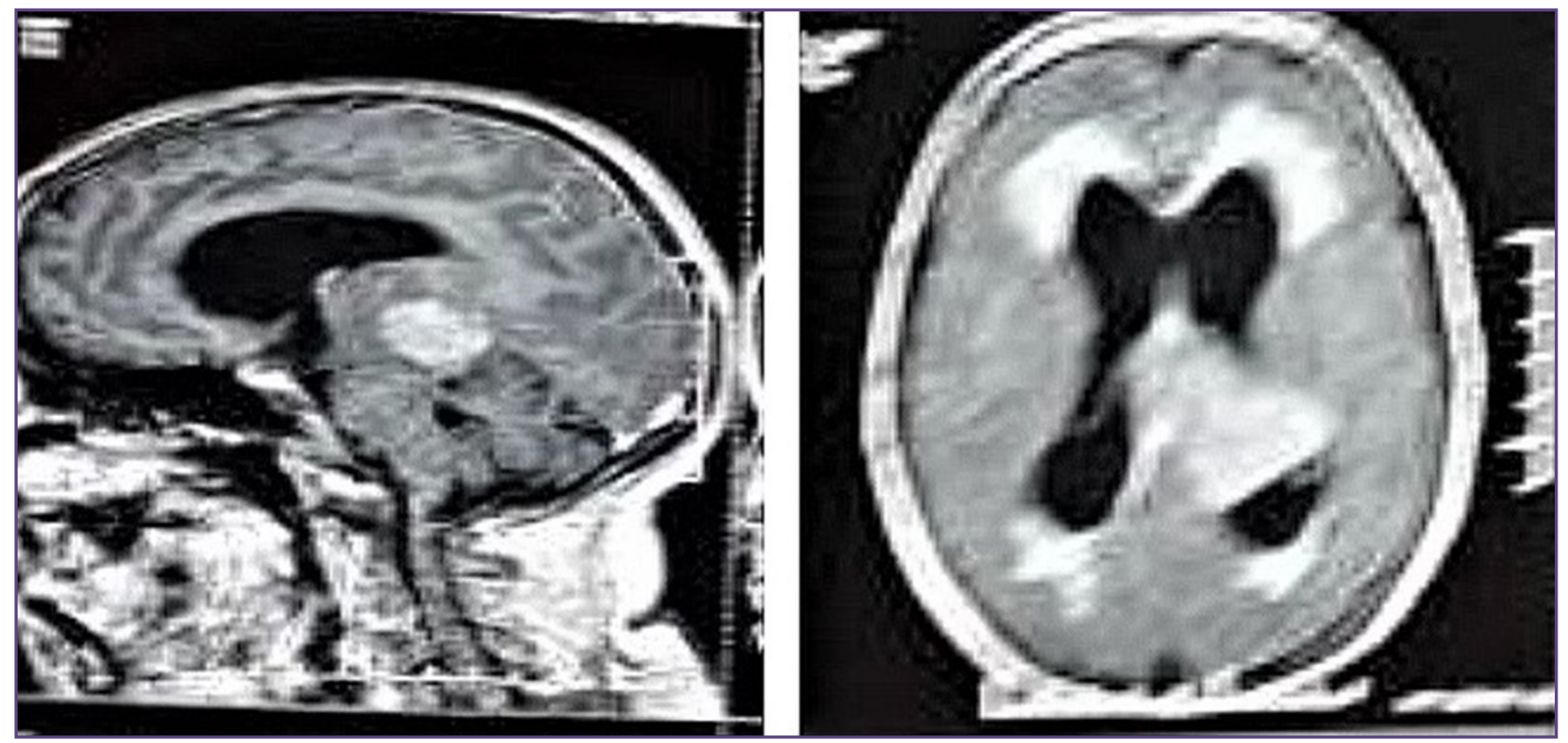

Fig. 1: MRI: T2 hyper intense. Infiltrative tumour $3 \times 3 \times 2 \mathrm{~cm}$ extending to pineal region.Inferior to the splenium of corpus callosum and above the cerebellar hemispheres.

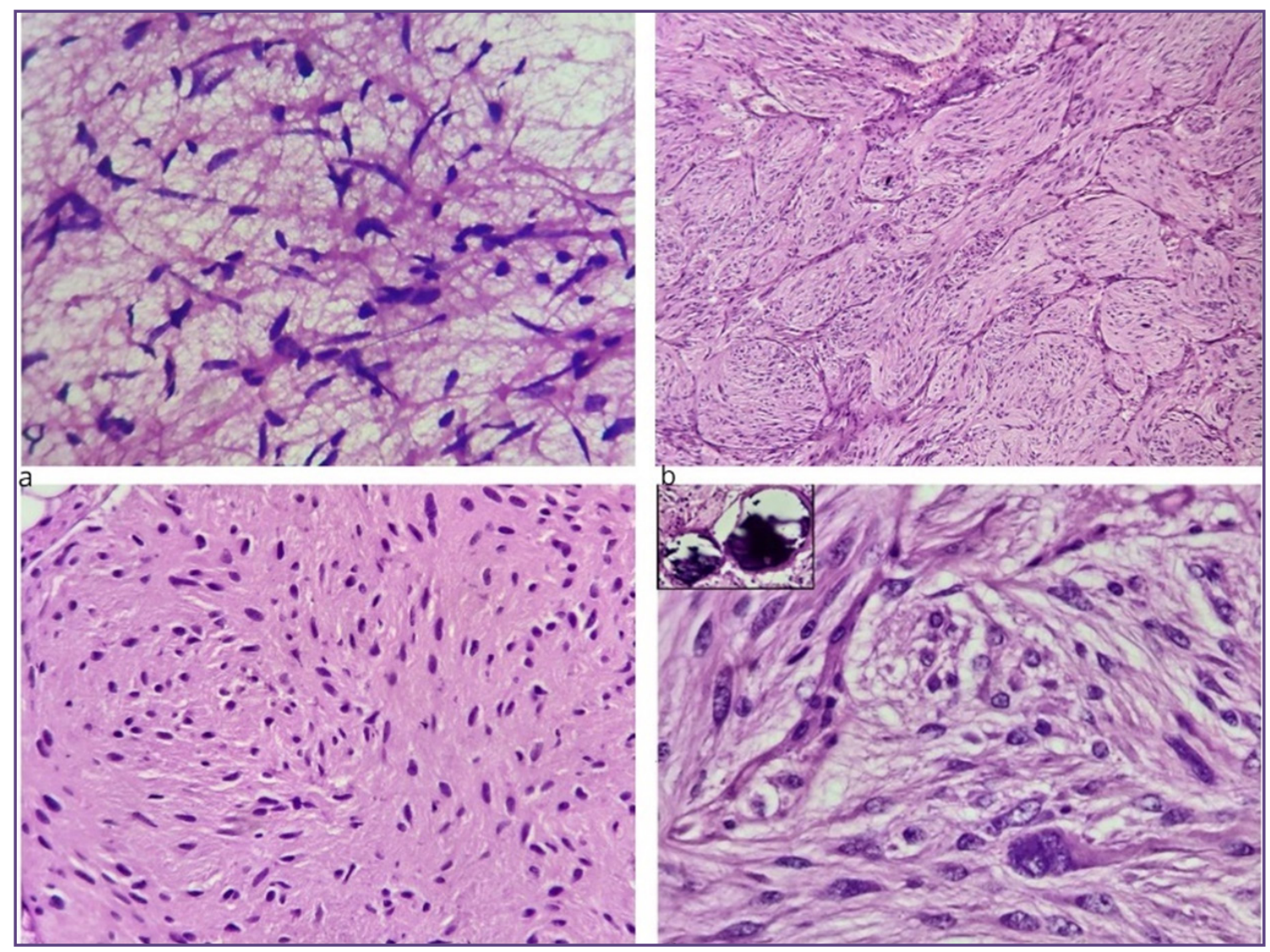

Fig. 2.a: Squash: Low grade glioma. (100x H\&E), b: Nodular (Plexiform)architecture (100x, H\&E), c: Low grade glial morphology with fibrillary background. (400x H\&E), d: Moderate nuclear pleomorphism with few large nuclei and areas of calcification.(400x H\&E) 


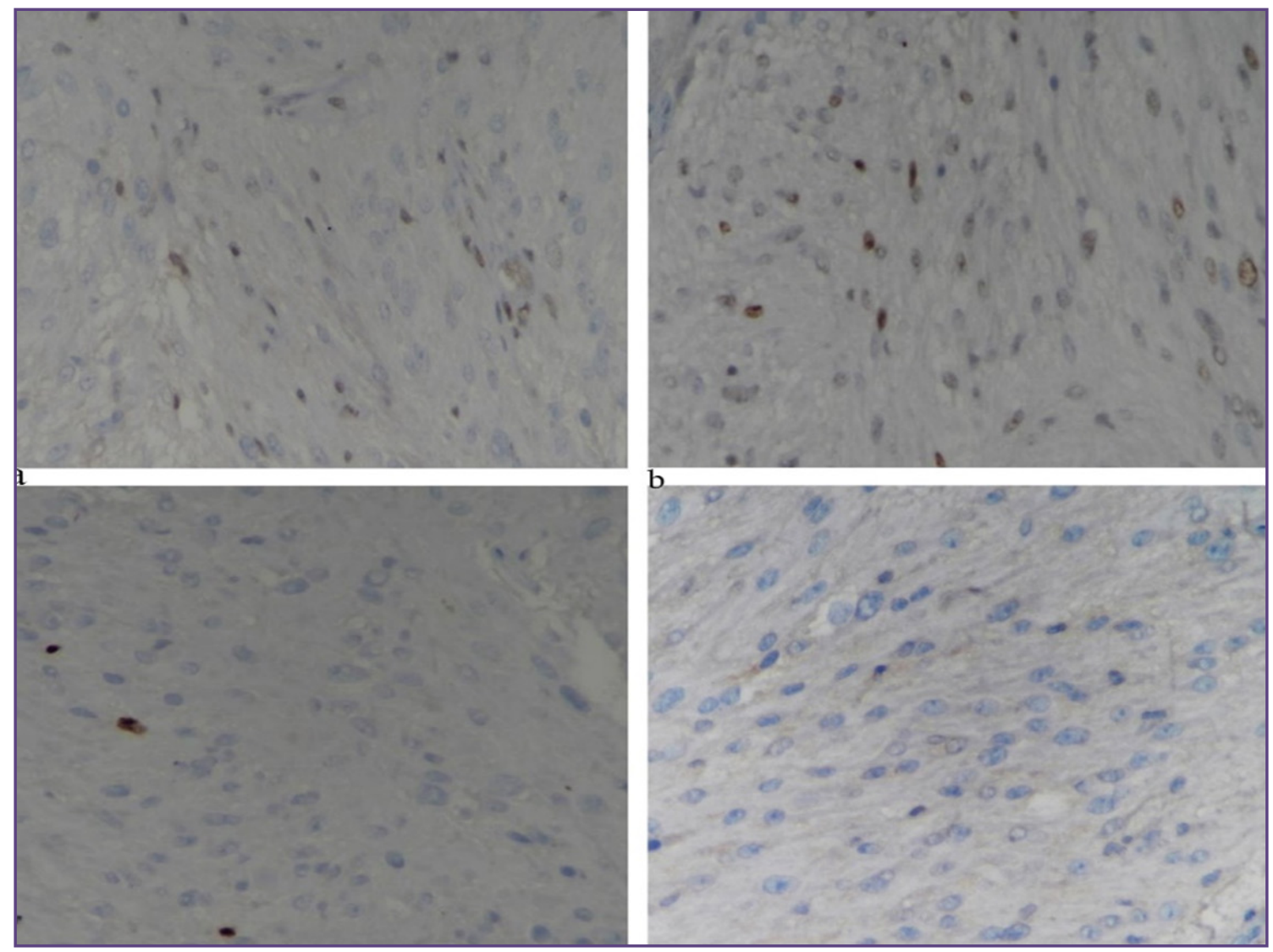

Fig. 3.a: Loss of ATRX protein, b: Focal positivity for p53 protein, c: Low MIB- 1 index(3-4\%), d: IDH1R132H negative..

\section{RJ27353 \\ 38393CR}

H3F3A K27M mutant

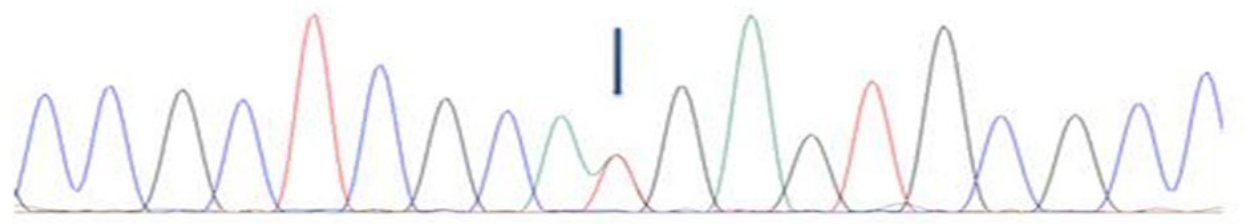

PRIMER OF H3F3A A > T MUTANT SHOWS TRANSVERSION TO METHIONINE (AAG TO ATA)

\section{HIST1HзB Wild type}

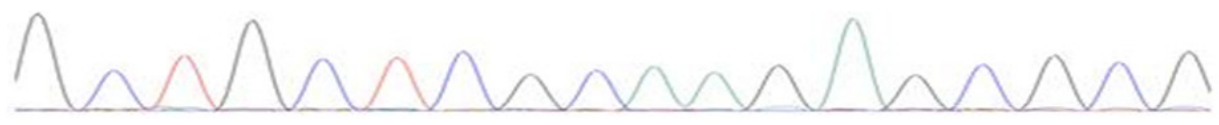

Fig. 4: H3K27M Mutation detected by Sanger sequencing. 
$40 \%$ showing enhancement. In contrast, paediatric Diffuse Intrinsic Pontine Glioma is non contrast enhancing. ${ }^{[3]}$

Histones are protein components that aredeposited during DNA synthesis. The nucleosome core is composed of an octamer ofH2A, H2B, H3 \& H4 and is linked to DNA via Histone H1.H3 has 3 isoforms 3.1,3.2 \&3.3 which are important for transcription, telomerase activity andDNA repair.DMG typically shows Gain of function mutations in H3 Histone genes. Histone 3A (H3F3A) encodes histone $\mathrm{H} 3$ variant $\mathrm{H} 3.3$ and Histone H3B (HIST1H3B), encodes histone H3 variant H3.1. Histone H3.3 mutations occur at two distinct residues, either lysine at position 27 or glycine at position 34 resulting in replacement of lysine 27 by methionine $(\mathrm{K} 27 \mathrm{M})$ or replacement of glycine 34 by valine $(\mathrm{G} 34 \mathrm{~V})$. Lysine to methionine substitution at codon 27 usually involves H3F3A but may also involve HIST1H3B/C genes (H3 K27M mutations) and is associated with aggressive diffuse midline gliomas. ${ }^{[4]}$

Several studies have reported that K27M mutant tumors are mostly seen in the midline structures whereas G34V mutant tumors occur in the cerebral hemispheres. ${ }^{[4,5]}$ High grade gliomas associated with Histone H3.1 (HIST1H3B) mutations are seen predominantly in neonates and pediatrics, are usually located in the pontine location, associated with ACVR 1, OLIG1 \&2 mutations and have a median survival of 15 months. In contrast, Histone H3.3(H3F3A) associated gliomas can occur at any age, predominantly occur in any midline location, associated with TP53, DAXX \& ATRX mutations and have a poor median survival of 9 months. ${ }^{[4,5]}$

Meyronet et al evaluated 21 adult patients with H3K27M mutant diffuse gliomas and found that all tumors had a midline location with $29 \%$ located in the spinal cord followed by $24 \%$ in the thalamus and $24 \%$ in the midbrain. Only 5\% tumors were located in the pineal region as in our case. The clinical presentation was variable and consisted of progressive sensorimotor deficits (67\%) followed by intracranial hypertension (29\%), ataxia (24\%) and cranial nerve injury (19\%). On MRI, contrast enhancement was present in $50 \%$ of the cases. These diffuse gliomas had varied histomorphological features suggestive of a low gradeglioma (15\%) to glioblastoma multiforme (52\%).28\% of the cases showed morphology that could not be categorized whereas pilocyticastrocytoma like features were noted in $2.5 \%$ of the cases and $2.5 \%$ showedganglioglioma like features. ${ }^{[2]}$ Pineal neoplasms are usually low grade astrocytomas and very few case reports of glioblastoma multiforme have been reported. ${ }^{[6]}$ In our case too, low grade astrocytic tumor like pilocytic astrocytoma was considered as a differential on morphology.
Solomon et al studied 47 cases of Diffuse midline glioma $\mathrm{H} 3 \mathrm{~K} 27 \mathrm{M}$ in both adult and pediatric patients and found that although a wide morphologic spectrum can be seen, all tumors show at least someclassic astrocytic morphology characterized by ovoid to elongated nuclei with coarse chromatin and widespread oligodendroglial morphology is almost never seen.He reported histomorphology varying from gliomas with giant cells, epithelioid and rhabdoid cells, primitive neuroectodermal tumor (PNET)-like foci, neuropil-like islands, pilomyxoid features, ependymallike areas, ganglionic differentiation to pleomorphic xanthoastrocytoma (PXA)-like areas. Majority of the tumors were centered in the pons, thalamus, and spinal cord, but a few were also located in the third ventricle, hypothalamus, pineal region and cerebellum ${ }^{[7]}$

Schreck evaluated 163 cases of midline gliomas of which $15 \%$ had H3K27M mutation, of these only one case had high grade (III/IV) histomorphology, rest were all low grade, as was seen in our case. The most common locations for $\mathrm{H} 3 \mathrm{~K} 27 \mathrm{M}$ mutated tumors were midbrain (67\%), pons (36\%) and cerebellum (25\%). Also, there was no difference in the contrast enhancement on MRI between H3K27M mutant tumors and wild type tumors. ${ }^{[8]}$

Adult midline gliomas predominantly show H3K27M mutation. They are frequently associated with loss of ATRX (46.8\%) and mutation of TP53(42.4\%). They almost never show IDH1, EGFR, TERT and BRAF V 600 E mutations. ${ }^{[7,8]}$

The median survival rate of these cases is 19.6 months as reported by Meyronet et al. ${ }^{[2]}$ Recent reports have proposed that the survival of these cases may be similar or possibly improved as compared to gliomas with wild type mutation, however this needs further validation. ${ }^{[8]}$

Identification of this $\mathrm{H} 3 \mathrm{~K} 27 \mathrm{M}$ mutation in diffuse midline gliomas is of utmost importance as it is has not only prognostic but also therapeutic significance. Various potential targeted treatment options are now under trial. Histone deacetylase inhibitor (HDACi), GSKJ4 has invitro and in-vivo anti-tumour activity against K27M mutant tumours. Vorinostat, a pan-HDACi has shown benefit in pre-clinical trials. Panobinostat (HDACi), has better activity than Vorinostat in-vitro. Trial of Panobinostat in Children with Diffuse Intrinsic Pontine Glioma (PBTC047) is currently open. ${ }^{[9]}$

In order to diagnose DMG $\mathrm{H} 3 \mathrm{~K} 27 \mathrm{M}$ mutant, a tumour should be diffuse, midline, a glioma and H3K27 M mutant, just presence of the mutation does not warranty a diagnosis of DMG(H3K27M) mutant. ${ }^{[10]}$ 


\section{Conclusion}

Diffuse midline glioma H3K27M mutant is a midline tumour that can have varied morphology on histopathology. Unlike other WHO grade IV tumours, it does not require the following criteria: atypia, mitosis, endothelial hyperplasia and necrosis for diagnosis. Any tumour with predominantly astrocytic differentiation and located in midline structures, should always be tested for H3K27M mutation.

Acknowledgements: Dr. Epari Sridhar, Tata memorial hospital, Mumbai for immunohistochemistry and genetic study

\section{Funding}

Nil

\section{Competing Interests}

Nil

\section{References}

1. David N Louis, Hiroko Ohgaki, Otmar D.Wiestler, Webster K. Cavenee (Eds): WHO Classification of Tumours of the Central Nervous System (Revised 4th edition). IARC; Lyon 2016.

2. Meyronet D, Esteban-Mader M, Bonnet C, Joly MO, Uro-CosteE, Amiel-Benouaich A et al. Characteristics of H3 K27M-mutant gliomas in adults: Neuro Oncol.2017;19:1127-1134.

3. $\mathrm{Hu} \mathrm{J}$, Western S, Kesari S. Brainstem Glioma in Adults. Front Oncol. 2016; 6: 180
4. LullaRR, Saratsis AM, HashizumeR. Mutations in chromatin machinery and pediatric high-grade glioma. Sci Adv.2016;2: e1501354.

5. Castel D, Philippe C, CalmonR, LeDret L, Truffaux N, Boddaert $\mathrm{N}$ et al. Histone H3F3A and HIST1H3B K27M mutations define two subgroups of diffuse intrinsic pontine gliomas with different prognosis and phenotypes.Acta Neuropathol.2015;130:815-27

6. Stowe HB, Miller CR, Wu J, Randazzo DM, Ju AW. Pineal Region Glioblastoma, a Case Report and Literature Review. Front. Oncol. 2017;12:123.

7. Solomon DA, Wood MD, Tihan T, Bollen AW, Gupta $\mathrm{N}$, Phillips $\mathrm{J}$ et al.Diffuse Midline Gliomas with Histone H3-K27M Mutation: A Series of 47 Cases Assessing the Spectrum of Morphologic Variation and Associated Genetic Alterations.Brain Pathol. 2016;26:569-80.

8. Schreck KC, Ranjan S, Skorupan N, Bettegowda $\mathrm{C}$, Eberhart $\mathrm{CG}$, Ames $\mathrm{HM}$ et al. Incidence and clinicopathologic features of $\mathrm{H} 3 \mathrm{~K} 27 \mathrm{M}$ mutations in adults with radiographically-determined midline gliomas. J Neuro-Oncol. 2019;143:87-93.

9. Grasso CS, Tang Y, Truffaux N, Berlow NE, Liu L, Debily MA et al. Functionally defined therapeutic targets in diffuse intrinsic pontine glioma. Nat Med. 2015;21:555-9.

10. Louis DN, Giannini C, Capper D, Paulus W, FigarellaBranger D, Lopes $\mathrm{MB}$ et al. cIMPACT-NOW update 2: diagnostic clarifications for diffuse midline glioma, H3 K27M-mutant and diffuse astrocytoma/ anaplastic astrocytoma, IDH-mutant. Acta Neuropathol. 2018;135:639-642.

*Corresponding author:

Dr.Sonal Paul, A-5/102, Chabhaiya Park, Kapurwadi, Thane (w)-400607

Phone: +91 9930856879

Email: sonalpaul1993@gmail.com

Date of Submission : 11/05/2020

Date of Acceptance : 05/09/2020

Financial or other Competing Interests: None.
Date of Publication : 30/10/2020 